\title{
The evaluation of gait problems and related factors in patients with Parkinson's disease
}

\begin{abstract}
Background/aim: The purpose of study is to determine the gait performance and other factors related to turning in mild and moderate phase Parkinson's disease patients (PD).

Methods: Ninety two patients were followed up for observation. After passing neurological examinations, the Unified Parkinson Disease Rating Scale (UPDRS), Hoehn/Yahr Scale (H\&Y), Mini Mental State Examination (MMSE), Tinetti test, Berg Balance test, $6 \mathrm{~m}$ walking test, step count, $360^{\circ}$ turning duration, and Functional Independence Measure (FIM) were applied. All cases were evaluated according to the "on" period.
\end{abstract}

Results: Forty one females and 51 males participated in the study. The duration of time it took to turn right was $8.22 \pm 6.26$ seconds, and the time it took to turn from left was $8.51 \pm 6.63 \mathrm{sec}$. There appeared to be no statistical difference in the turn times of patients either in terms of the socio-economic factors of gender, education level, or employment status. But, statistically significant relationships were found when it came to in both directions, age, duration of illness, UPDRS, FIM, Tinetti, the Berg Test, the walking test, and the number of steps taken.

Conclusion: Results suggest that 360 degree turning performance in mild and moderate stage PD is strongly associated with patient's age, disease duration and disease severity.

Keywords: parkinson's disease, gait, factors, ignition failure, postural instability, freeze
Volume 2 Issue 3 - 2018

\author{
Ferhan Soyuer,' Murat Gultekin, ${ }^{2}$ Feyzan \\ Cankurtaran' \\ 'Department of Physiotherapy and Rehabilitation, Nuh \\ Naci Yazgan University, Turkey \\ ${ }^{2}$ Department of Neurology, Erciyes University Faculty \\ of Medicine, Turkey
}

Correspondence: Ferhan Soyuer, Department of Physiotherapy and Rehabilitation, Nuh Naci Yazgan University, Faculty of Health Sciences, Kayseri /Turkey, Tel 05422354062, Email soyuerferhan@gmail.com

Received: May 17, 2018 | Published: June 04, 2018
Abbreviations: UPDRS, unified parkinson disease rating scale; FIM, functional independence measure; PD, parkinson's disease; UK-PDSB, united kingdom parkinson's disease society brain bank; MMSE, mini mental state examination

\section{Introduction}

Parkinson's disease (PD) usually occurs over the age of 60 and is the most common of Parkinsonism disorders. It is characterized by damage of dopamine-producing cells located in substansiya nigra region of the brain. It is characterized by the presence of cytoplasmic Lewy inclusion bodies. The cause of nigral degeneration which are responsible of the development of this disease is currently unknown., ${ }^{1,2}$ PD is a progressive disease, and its symptoms include bradykinesia, resting tremors, cogwheel rigidity, and postural instability. ${ }^{3}$ Postural and gait disorders that occur within this disease play an important role in causing severe disability. ${ }^{4}$ As gait pattern in Parkinson's disease differs at various stages of the disease, and its effects vary from patient to patient. The shortening of step length as well as the decrease in number of steps per minute ("cadence") is most typically observed. Thus patients shamble and walk slowly with small-steps. Common stages in advanced stages of the disease include the hassle of trying to start to walk ("ignition failure"), stoping while walking (motor blocks), postural imbalance and difficulty turning. ${ }^{5,6}$ Parkinson's gait is slow, it covers a narrow area, and is characterized by small steps whereby the patient's feet drag-in so far that those observing such patients from distance assume that the patient losing his or her centre of gravity. Patients lose their ability to fully swing their arms, they become slow at rotating, it takes them several small steps in order to be able to turn, and at times they freeze.

This can be observed in especially narrow and crowded places. In some cases, it is possible to observe the rapid steps in succession, whereby the patient's walking pattern (festination) is halted when they encounter a barrier, thus making it difficult for them to walk and turn. More than $50 \%$ of PD patients have been affected by turnover disease, which is usually observed before other gait abnormalities. ${ }^{7}$ Difficulty in turning around the axial axes is a common and early feature of PD, and can be present when lying down, sitting, standing, or walking. Turning difficulties affects daily activities, and is known to have a direct link with falling. This, in essence, significantly affects quality of life. ${ }^{8}$ Although patients with late stage PD are known to experience turning difficulties, little is known about the same problem when it comes to patients with mild and moderate forms of the disease, including the potential variables causing and affecting it. The aim of the study is to determine the turn times in patients with mild to moderate PD, as well as to determine the variables related to turning performance.

\section{Methods}

Ninety-two Parkinson's patients (51 males, 41 females) who were followed-up for at least 1 year at the Movement Disorders Clinic at XXXXX University's Department of Neurology between November 2015 and December 2016 were enrolled in this study. With the decision of XXXXX Ethical Committee of Clinical Research dated 17.9.2015 and 2015/414, patients with mild and moderate Parkinson's disease, 
and who are in line with the "United Kingdom Parkinson's Disease Society Brain Bank (UK-PDSB)" criteria in terms of having a normal or mild degree of cognitive decline, being able stand independently, having no incurable visual, hearing, and/or speech problems, and having no other orthopaedic, systemic, or neurological disease at the time, as well as who voluntarily agree to participate were involved in the study. The consent form was obtained from the patients. All cases were evaluated during the "on" (drug active) period. Patients were provided with information about the to be tests applied, and their written consent was obtained. First, neurological examinations were performed on all of the volunteers, followed by a battery of tests including the Unified Parkinson's Disease Rating Scale (UPDRS), Hoehn/Yahr staging, and Mini Mental State Examination (MMSE). The Tinetti test, Berg balance scale, $6 \mathrm{~m}$ walking test, Functional Independence Scale, and Turkish Neurological Society balance and gait disturbance evaluation form were used in this study.

\section{The mini mental state examination}

MMSE was used to assess the cognitive status of patients. They are grouped under five main categories: orientation (10 points), recording memory ( 3 points), attention and calculation ( 5 points), reminding ( 3 points), and language (9 points). This test consists of 11 items, and is evaluated out of a total of 30 points. In terms of results, a score of 27- 30 points means a normal border; a score of $24-26$ points means mild cognitive impairment, and a score of $<24$ points entails severe cognitive impairment.

\section{Hoehn/yahr staging}

The staging of the Parkinson's disease was done using the HoehnYahr scale. The Hoehn-Yahr scale examines the disease in terms of 5 stages. ${ }^{9}$

\section{The unified parkinson's disease assessment scale (UPDRS)}

The Unified Parkinson's Assessment Scale was used to determine the clinical severity of Parkinson's disease, where by the following criterion were used for statistical analysis: 1) Mental, behavioursal, and psychological status (16 points); 2) daily activities (52 points); 3-Motor sub-scores (92 points); the Total score of UPDRS.

\section{Walking}

It was tested in a 10-meter-long flat zone in polyclinic corridor. Values were recorded in the 6-meter walking area as well as in the middle section of the 10-meter walking range in all tests in order to exclude the effects of acceleration and deceleration at both the beginning (first 2 meters) and the end of the test (last 2 meters)

\section{The walking time (sec)}

Walking of the 6-meter distance was recorded using a chronometer, while another officer noted how many steps the patient took at the same distance. The mean values of the two tests were taken, whereupon the number of steps and walking time $(\mathrm{sec})$ at 6 meters was then calculated. ${ }^{10}$

\section{In observing 360-degree clockwise and counter- clockwise turnaround times}

Patients were asked to start turning and the360-degree rotation was completed and measured using a stopwatch. Rest breaks for the convenience of the patients were given between each rotation.

\section{The functional independence measure (FIM)}

This evaluates 18 activities, and consisting of 6 main parts including self-care, sphincter control, transfer, movement, communication, and social perception. Each activity is assessed in terms of functional independence using a 7-point scale, with the total score varying between 18 and 126. The assessment of communication and social perception was labelled as FIM cognitive, were as the evaluation of the other subgroups was labelled as FIM motor.

\section{The tinetti test}

This test is based on the evaluation of the patient during activities scored according to pre-determined qualitative criteria involving walking and equilibrium subsystems. Each activity is evaluated according specific scoring criteria, whereby the total score is a maximum of 28 with a walking subscale score $^{12}$ and a balance subscale score. ${ }^{16}$ It has been reported that elderly individuals living in the community have high predictive values when it comes to the risk of falling and associated injuries. ${ }^{11}$

\section{The berg balance scale}

This was used in order to evaluate balance in a validated and reliable manner, and assesses the ability of individuals to carry out balance while performing functional activities ${ }^{12}$ This test consists of 14 items, with each section being graded between 0 (worst) and 4 (best), and depends on positions such as standing up without standing, feet standing contiguous, standing in tandem position, standing on one leg, and/or the level of independence, and the person's ability to change positions. The highest score obtained from the BBS shows the best balance. According to the scores obtained from this test, the cases were divided into three groups: "high risk of falling (0-20 points)", "moderate risk of falling (21-40 points)", and "low risk of falling (4156 points)".

\section{Statistical analysis}

Statistical analysis of the study was done using the SPSS 22 statistical software package. Continuous variables were determined using the Shapiro Wilk test, which shows whether or not the variables show normal distribution. Descriptive statistics were given as number, percentage, and mean \pm standard deviation according to the variable types and distribution structure. Parametric tests were applied to variables with normal distribution. The variance analysis was used to test hypotheses whether or not the difference between the means of two or more groups was significant in any way. The Pearson correlation coefficient was calculated upon evaluating the relationship between variables. The $\mathrm{p}<0.05$ was considered for statistical significance.

\section{Results}

In terms of patient profiles, forty one (44.6\%) women and 51 $(55.4 \%)$ men participated in the study. Twenty $(21.7 \%)$ of the patients were not literate, $72(78.3 \%)$ had either a primary school-level of education or greater, $38(41.3 \%)$ were housewives, and $54(58.7 \%)$ were either retired or still working. The mean age of the participants was $61.65 \pm 12.32(29-88)$ years, and their duration of illness was $6.24 \pm 5.72$ (1-25) years (Table 1). According to the H\&Y stage scale, the patient group in this study (1-3) had either mild or moderate Parkinson's disease. The duration of turning of IPD 360 degrees from the right (clockwise) was $8.22 \pm 6.26(2-42)$ seconds, and the time of turning 360 degrees from left (counter-clockwise) was $8.51 \pm 6.63$ (2$45)$ sec. There was no statistical differences in turn times from both 
directions when it came to the patient's gender and employment status ( $p>0.05)$ (Table 2). There appeared to be significant statistical correlations between turning in both directions, and age $(p<0.01)$, duration of illness ( $p<0.01-0.02)$, the mental UPDRS, UPDRS-DLA,
UPDRS motor, UPDRS total, FIM, Tinetti walking-balance and its total, the Berg balance, $6 \mathrm{~m}$ walking time, and the number of steps taken $(\mathrm{p}<0.00)($ Table 3$)$.

Table I Sociodemographic and Clinical Variables of Patients with Parkinson's disease

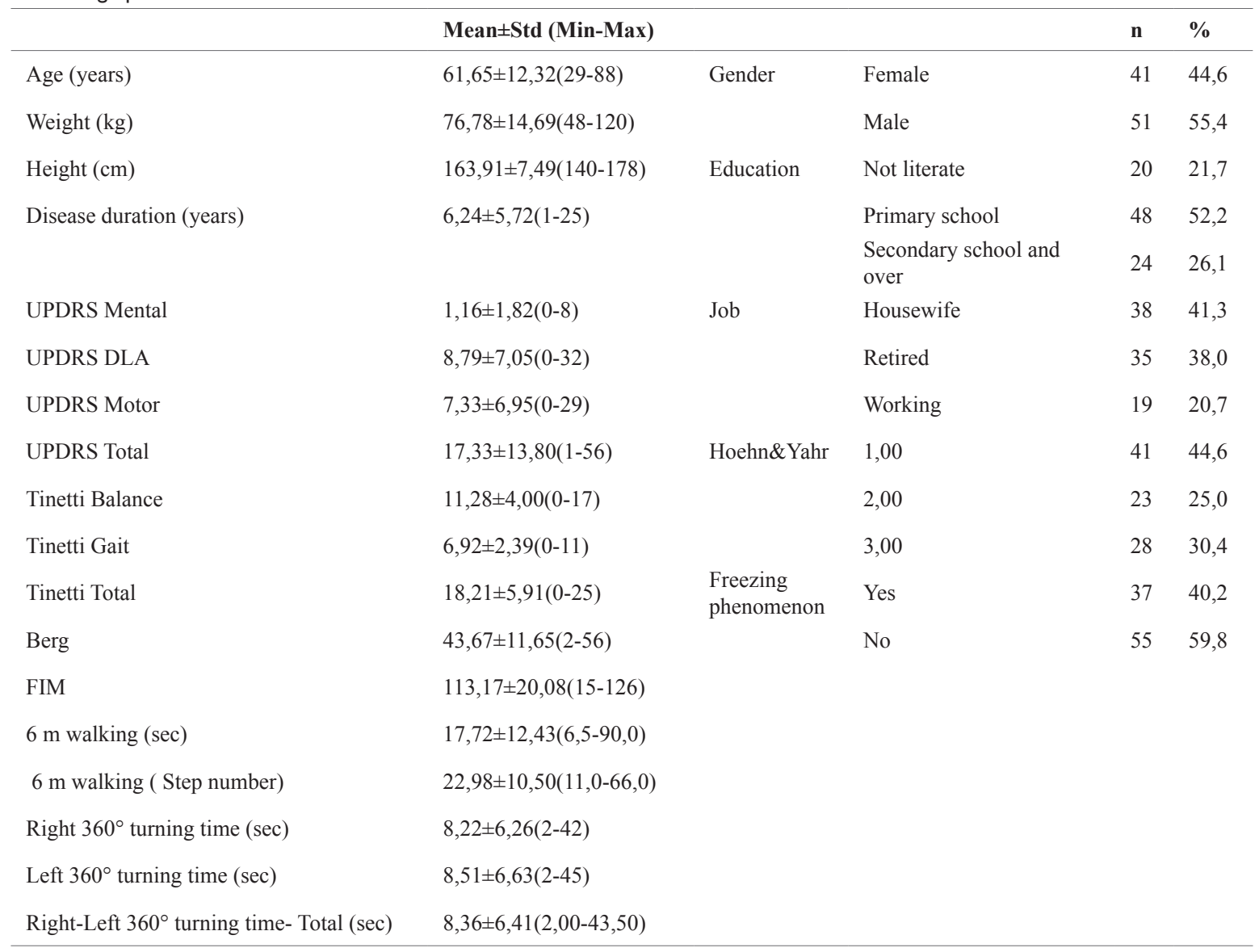

Table 2 Sociodemographic Variables According to Turning in Patients with Parkinson's disease

\begin{tabular}{|c|c|c|c|c|c|c|c|c|c|c|c|c|c|c|c|c|}
\hline & & \multicolumn{5}{|c|}{ Right $360^{\circ}$ turning time } & \multicolumn{5}{|c|}{ Left $360^{\circ}$ turning time } & \multicolumn{5}{|c|}{ Right-left $360^{\circ}$ turning time- Total } \\
\hline & & $\mathbf{N}$ & $\mathbf{X} \pm \mathbf{S S}$ & $\mathbf{F}$ & $\mathbf{t}$ & $\mathbf{p}$ & $\mathbf{N}$ & $\mathbf{X} \pm \mathbf{S S}$ & $\mathbf{F}$ & $\mathbf{t}$ & $\mathbf{p}$ & $\mathbf{N}$ & $\mathbf{X} \pm \mathbf{S S}$ & $\mathbf{F}$ & t & $\mathbf{p}$ \\
\hline \multirow{3}{*}{ 空 } & Female & 41 & $8,67 \pm 6,16$ & & 620 &, 537 & 41 & $9,04 \pm 6,39$ & & ,686 & ,494 & 41 & $8,85 \pm 6,24$ & & ,658 &, 513 \\
\hline & Male & 51 & $7,85 \pm 6,37$ & & & & 51 & $8,08 \pm 6,85$ & & & & 51 & $7,96 \pm 6,58$ & & & \\
\hline & $\begin{array}{l}\text { Not } \\
\text { literatel** }\end{array}$ & 20 & $9,78 \pm 6,47$ & & & & 20 & $10,78 \pm 7,18$ & & & & 20 & $10,27 \pm 6,80$ & & & \\
\hline \multirow{4}{*}{ 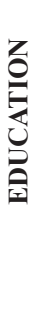 } & $\begin{array}{l}\text { Primary } \\
\text { school }\end{array}$ & 48 & $8,71 \pm 6,94$ & 2,431 & & ,094 & 48 & $8,85 \pm 7,13$ & 3,212 & &, 045 & 48 & $8,78 \pm 7,00$ & 2,827 & &, 065 \\
\hline & $\begin{array}{l}\text { Secondary } \\
\text { school and } \\
\text { over ** }\end{array}$ & 24 & $5,94 \pm 3,76$ & & & & 24 & $5,92 \pm 3,97$ & & & & 24 & $5,92 \pm 3,84$ & & & \\
\hline & Housewife & 38 & $8,67 \pm 6,28$ & 1,867 & & ,161 & 38 & $9,12 \pm 6,51$ & 1,380 & &, 257 & 38 & $8,89 \pm 6,36$ & 1,611 & & ,205 \\
\hline & & 35 & $9,04 \pm 6,98$ & & & & 35 & $9,06 \pm 7,538$ & & & & 35 & $9,05 \pm 7,23$ & & & \\
\hline$\stackrel{\oplus}{\varrho}$ & Working & 19 & $5,79 \pm 4,12$ & & & & 19 & $6,26 \pm 4,605$ & & & & 19 & $6,02 \pm 4,34$ & & & \\
\hline
\end{tabular}

**A significant difference was found in terms of $360^{\circ}$ turning time from the left between the non-illiterate and secondary school and over. 
Table 3 Pearson Correlations Between Variables

\begin{tabular}{|c|c|c|c|c|c|c|}
\hline & \multicolumn{2}{|c|}{ Right $360^{\circ}$ turning time } & \multicolumn{2}{|c|}{ Left $360^{\circ}$ turning time } & \multicolumn{2}{|c|}{ Right-left $360^{\circ}$ turning time- Total } \\
\hline & $\mathbf{r}$ & $\mathbf{p}$ & $\mathbf{r}$ & $\mathbf{p}$ & $\mathbf{r}$ & $\mathbf{p}$ \\
\hline Age (years) & ,338 &, 001 & ,315 & ,002 &, 328 &, 001 \\
\hline Weight (kg) &,- 067 &, 527 &,- 119 &, 260 &,- 094 & ,373 \\
\hline Height (cm) &, 241 &, 020 &, 267 &, 010 &, 256 &, 014 \\
\hline Disease duration (years) & ,407 &, 000 & ,441 &, 000 &, 427 &, 000 \\
\hline UPDRS DLA &, 535 &, 000 &, 576 &, 000 &, 559 &, 000 \\
\hline UPDRS Motor &, 437 &, 000 & ,463 &, 000 & ,453 &, 000 \\
\hline UPDRS Total &, 547 &, 000 &, 585 &, 000 &, 569 &, 000 \\
\hline Tinetti Total &,- 632 &, 000 &,- 657 &, 000 &,- 648 &, 000 \\
\hline Berg &,- 719 &, 000 &,- 725 &, 000 &,- 725 &, 000 \\
\hline FIM &,- 394 &, 000 &,- 397 &, 000 &,- 398 & 0 \\
\hline $6 \mathrm{~m}$ walking (sec) &, 575 &, 000 &, 571 &, 000 &, 575 &, 000 \\
\hline $6 \mathrm{~m}$ walking (Step number) & 627 &, 000 & ,626 &, 000 & ,629 &, 000 \\
\hline
\end{tabular}

\section{Discussion}

Study showed that 360-degree right and left turn times and age are significantly related to disease severity, balance, walking and functioning in PD patients. In Parkinson's disease, difficulty in turning is a common problem and is associated with the phenomena of falling and freezing. ${ }^{5}$ Falling during turning was reported to be 8 times greater than falling during walking, and in turn caused hip fractures. Laboratory studies on patients with moderate and severe PD show that during the act of turning, velocity decreases, the number of steps increases, the time of the turning increases, the support surface narrows, and the rotten segmented co-ordination (en-bloc) is detected. However, found malfunctions in the turning characteristics of mildly ill patients who lack walking deficits. ${ }^{13}$ study also showed that mild and medium stage PD had a longer 360 degree turning period and a decreased rate of speed. In healthy people, a turning accompanied by a cranio-caudal line followed by the head, body, and pelvis is observed, whereas in Parkinson's patients, turning tends to first be performed with the head and body turning together, and then is followed by the pelvis. In healthy people, rotating from right and left shows similar features. However, in Parkinson's patients there are differences in the number of steps, rate of speed, and turn qualities when rotating either from the left or from the right. In our study, turn from left appeared to be longer in the PD patients we observed. There is no relationship between rotation and the laterality of symptoms, or turning from the more affected side documented in current medical literature. ${ }^{14}$

In research, we have determined that the turnaround time increases as the duration of illness extends. As the disease progresses, it is known that walking and balance problems more frequently accompany the disease. The data in study also supports the occurrence of these problems over the course of the progression of the disease. Anti-parkinson drug therapy has no effect on turning performance (duration, position of body segments, etc.) in Parkinson's patients. ${ }^{15,16}$ This finding is in line with our study in that drug treatment appears to have no effect on axial movements. In our studies we have evaluated the re-turn of patients from the right and left at 45 to 90,135 , and 180 degrees. ${ }^{17-19}$ In study, 360 degree turning performance was evaluated in PD for the first time. This motion was looked at because it is an activity that we often use in daily activities. There were no statistically significant difference in terms of and between socio-demographic variables, with the exception age and how that impacts the PD ability to turn 360 degrees clockwise or counter-clockwise. There currently is no study in the literature describing the relationship between turning and sociodemographic variables. As the $6 \mathrm{~m}$ walking time and the number of steps increased, the turn times had also increased rather dramatically. Likewise, as the UPDRS mental values had become displaced, the turnover duration of the patients had increased, deficiencies in daily activities as assessed by the FIM had increased, and the turnover duration of the patients had increased upon walking values of Tinnitus test losing order. In terms of these variables, study provides medical and scientific literature with a first-ever glimpse at this specific subject.

Turning difficulty is common among people with PD in advanced stages. People with PD turn slowly with prominent small steps, even demonstrating gait disturbances such as freezing of gait. ${ }^{20-23}$ But study has shown that there is a problem of turning in mild to moderate Parkinson's disease. It also showed that in these patients, the ability to turn was associated with age, duration of illness, UPDRS, FIM, Tinetti walking-balance, the Berg balance, $6 \mathrm{~m}$ walking time, and the number of steps taken. We have a few limitations in this study. The aim of this study was to determine the most effective variables involved in turning performance. However, further analysis was not possible because statistically significant correlations were not found between these variables. A distinctive feature of the protocol adopted in this study was that turning ability was assessed in patients with mild stage. As a general comment, most of PD patients had relatively low H\&Y stages, and the inclusion of PD patients with more advanced disease stages might have led to different results.

\section{Conclusion}

In conclusion, results suggest that 360 degree turning performance in mild and moderate stage PD is strongly associated with patient's age, disease duration and disease severity. Future studies are suggested to consider different turning scenarios, including different turning angles and different turning arcs. 


\section{Acknowledgements}

None.

\section{Conflict of Interest}

No conflict of interest was declared by the authors.

\section{References}

1. Titova N, Padmakumar C, Lewis SJ, et al. Parkinson's: a syndrome rather than a disease? J Neural Transm. 2016;124(8):1667-1676.

2. Uem JM, Marinus J, Canning C, et al. Health-Related Quality of Life in patients with Parkinson's disease--A systematic review based on the ICF model. Neurosci Biobehav Rev. 2016;61:26-34.

3. Raggi A, Leonardi M, Ajovalasit D, et al. Disability and profiles of functioning of patients with Parkinson's disease described with ICF classification. Int J Rehabil Res. 2011;34(2):141-150.

4. Doherty KM, Warrenburg BP, Peralta MC, et al. Postural deformities in Parkinson's disease. Lancet Neurol. 2011;10(6):538-549.

5. Hulbert S, Ashburn A, Robert L, et al. A narrative review of turning deficits in people with Parkinson's disease. Disabil Rehabil. 2015;37(15):1382-1389.

6. Benatru I, Vaugoyeau M, Azulay JP. Postural disorders in Parkinson's disease. Neurophysiol Clin. 2008;38(6):459-465.

7. Morris ME, Huxham F, McGinley J, et al. The biomechanics and motor control of gait in Parkinson disease. Clin Biomech (Bristol, Avon). 2001;16(6):459-470.

8. Akram S, Frank JS, Jog M. Parkinson's disease and segmental coordination during turning: II. Walking turns. Can J Neurol Sci. 2013;40(4):520-526.

9. Hoehn MM, Yahr MD. Parkinsonism: Onset, progression and mortality. Neurology. 1967;17(5):427-442.

10. Allison L, Fuller K, Balance and vestibular disorders. In: Umphred DA, editor. Neurological Rehabilitation. Mosby. New York: 2001. 616-660 p.

11. Tinetti ME. Performance oriented assessment of mobility problems in the elderly patient. Am Geriatr Soc. 1986;34(2):119-126.
12. Qutubuddin AA, Pegg PO, Cifu DX, et al.Validating the Berg Balance Scale for patients with Parkinson's disease: a key to rehabilitation evaluation. Arch Phys Med Rehabil. 2005;86(4):789-792.

13. Carpinella I, Crenna P, Calabrese E. Locomotor function in the early stage of Parkinson's disease. IEEE Trans neural Syst Rehabil eng. 2007;15:543-551.

14. Stack E, Ashburn A. Dysfunctional turning in Parkinson's disease. Disabil Rehabil. 2008;30(16):1222-1229.

15. Hong M, Earhart GM. Effects of medication on turning deficits in individuals with Parkinson's disease. J neurol Phys ther. 2010; 34(1):11-16.

16. Hong M, Perlmutter JS, Earhart GM.Akinematic and electromyographic analysis of turning in people with Parkinson disease. Neurorehabil neural Repair. 2009;23(2):166-176.

17. Anastasopoulos D, Ziavra N, Savvidou E, et al. Altered eye-to-foot coordination in standing parkinsonian patients during large gaze and whole-body reorientations. Mov Disord. 2011;26(12):2201-2211.

18. Anastasopoulos D, Ziavra N, Hollands M, et al. Gaze displacement and inter-segmental coordination during large whole body voluntary rotations. Exp brain Res. 2009;193(3):323-336.

19. Haertner L, Elshehabi M, Zaunbrecher L, et al. Effect of Fear of Falling on Turning Performance in Parkinson's Disease in the Lab and at Home. Front Aging Neurosci. 2018;10:78-82.

20. Son M, Youm C, Cheon S, et al. Evaluation of the turning characteristics according to the severity of Parkinson disease during the timed up and go test. Aging Clin Exp Res. 2017;29(6):1191-1199.

21. GhangHong Y, MyeoungGon L, YouKyung K. Turning Characteristics In Patients With Parkinson's Disease During Timed Up And Go. 34. International Conference on Biomechanics in Sports. 2016.

22. Crenna P, Carpinella I, Rabuffetti M, et al. The association between impaired turning and normal straight walking in Parkinson's disease. Gait Posture. 2007;26(2):172-178.

23. Yang WC, Hsu WL, Wu RM, et al. Motion analysis of axial rotation and gait stability during turning in people with Parkinson's disease. Gait Posture. 2016;44:83-88. 